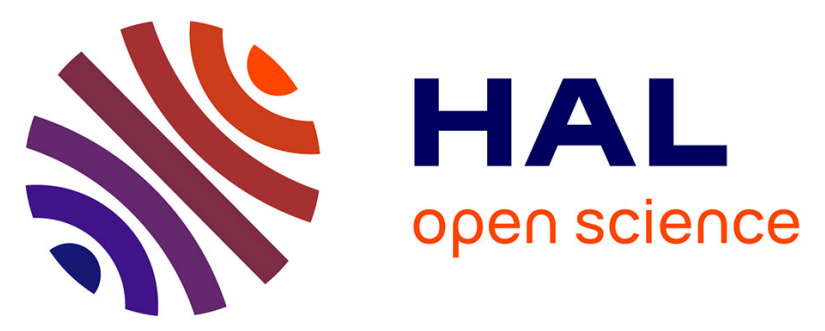

\title{
Secretogranin II is overexpressed in advanced prostate cancer and promotes the neuroendocrine differentiation of prostate cancer cells
}

Maité Courel, Fatima-Zohra El Yamani, David Alexandre, Hinde El Fatemi, Charlène Delestre, Maite Montero-Hadjadje, Fadl Tazi, Afaf Amarti, Rabia Magoul, Nicolas Chartrel, et al.

\section{To cite this version:}

Maïté Courel, Fatima-Zohra El Yamani, David Alexandre, Hinde El Fatemi, Charlène Delestre, et al.. Secretogranin II is overexpressed in advanced prostate cancer and promotes the neuroendocrine differentiation of prostate cancer cells. European Journal of Cancer, 2014, 50 (17), pp.3039-3049. 10.1016/j.ejca.2014.09.009 . hal-02341807

\section{HAL Id: hal-02341807}

https://hal-normandie-univ.archives-ouvertes.fr/hal-02341807

Submitted on 4 Nov 2019

HAL is a multi-disciplinary open access archive for the deposit and dissemination of scientific research documents, whether they are published or not. The documents may come from teaching and research institutions in France or abroad, or from public or private research centers.
L'archive ouverte pluridisciplinaire HAL, est destinée au dépôt et à la diffusion de documents scientifiques de niveau recherche, publiés ou non, émanant des établissements d'enseignement et de recherche français ou étrangers, des laboratoires publics ou privés. 


\title{
Secretogranin II is overexpressed in advanced prostate cancer and promotes the neuroendocrine differentiation of prostate cancer cells
}

\author{
Maïté Courel $^{\mathrm{a}, \mathrm{b}, 1}$, Fatima-Zohra El Yamani ${ }^{\mathrm{c}}$, David Alexandre ${ }^{\mathrm{a}, \mathrm{b}}$, Hinde El Fatemi ${ }^{\mathrm{d}}$, \\ Charlène Delestre ${ }^{\mathrm{a}, \mathrm{b}}$, Maité Montero-Hadjadje ${ }^{\mathrm{a}, \mathrm{b}}$, Fadl Tazi $^{\mathrm{d}}$, Afaf Amarti ${ }^{\mathrm{d}}$, \\ Rabia Magoul ${ }^{\mathrm{c}}$, Nicolas Chartrel ${ }^{\mathrm{a}, \mathrm{b}}$, Youssef Anouar ${ }^{\mathrm{a}, \mathrm{b}, *}$
}

\footnotetext{
a INSERM U982, Laboratory of Neuronal and Neuroendocrine Differentiation and Communication, Institute for Research and Innovation in Biomedicine, University of Rouen, 76821 Mont-Saint-Aignan, France

${ }^{\mathrm{b}}$ Normandy University, Normandy, France

${ }^{\mathrm{c}}$ Laboratory of Neuroendocrinology and Nutritional and Climatic Environment, Faculty of Sciences Dhar-El Mahraz, University Sidi Mohamed Ben Abdellah, $30000 \mathrm{Fez}$, Morocco

${ }^{\mathrm{d}}$ Department of Pathology, University Hospital Hassan II, University Sidi Mohamed Ben Abdellah, 30000 Fez, Morocco
}

Received 16 May 2014; received in revised form 11 August 2014; accepted 15 September 2014

Available online 9 October 2014

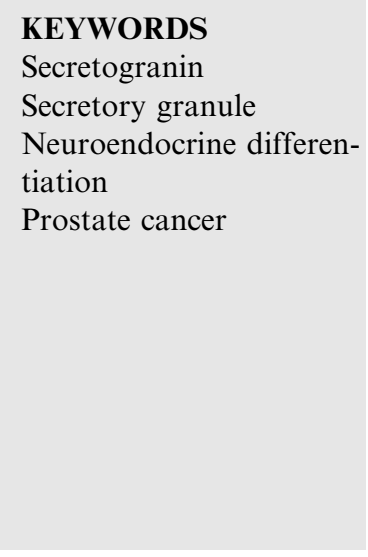

\begin{abstract}
Aim: In prostate cancer (PCa), neuroendocrine differentiation (NED) is commonly observed in relapsing, hormone therapy-resistant tumours after androgen deprivation. However, the molecular mechanisms involved in the NED of PCa cells remain poorly understood. In this study, we investigated the expression of the neuroendocrine secretory protein secretogranin II (SgII) in PCa, and its potential involvement in the progression of this cancer as a granulogenic factor promoting NED.

Methods: We have examined SgII immunoreactivity in 25 benign prostate hyperplasia and 32 $\mathrm{PCa}$ biopsies. In vitro experiments were performed to investigate the involvement of SgII in the neuroendocrine differentiation and the proliferation of PCa cell lines.

Results: We showed that immunoreactive SgII intensity correlates with tumour grade in PCa patients. Using the androgen-dependent lymph node cancer prostate cells ( $\mathrm{LNCaP}$ ) cells, we found that NED triggered by androgen deprivation is associated with the induction of SgII expression. In addition, forced expression of $\mathrm{SgII}$ in $\mathrm{LNCaP}$ cells implemented a regulated secretory pathway by triggering the formation of secretory granule-like structures competent
\end{abstract}

\footnotetext{
* Corresponding author at: INSERM U982, Laboratory of Neuronal and Neuroendocrine Differentiation and Communication, Institute for Research and Innovation in Biomedicine, University of Rouen, 76821 Mont-Saint-Aignan, France. Tel.: +33 235156661 ; fax: +33 235146946. E-mail address: youssef.anouar@univ-rouen.fr (Y. Anouar).

${ }^{1}$ Current address: CNRS-UMR7622, Institut de Biologie Paris-Seine, 7 quai Saint Bernard, 75252 Paris Cedex 05, France.
} 
for hormone storage and regulated release. Finally, we found that SgII promotes prostate cancer $(\mathrm{CaP})$ cell proliferation.

Conclusion: The present data show that SgII is highly expressed in advanced PCa and may contribute to the neuroendocrine differentiation by promoting the formation of secretory granules and the proliferation of PCa cells.

(c) 2014 Elsevier Ltd. All rights reserved.

\section{Introduction}

Neuroendocrine differentiation (NED) is a common dynamic feature of prostate cancer $(\mathrm{PCa})$, one of the most frequent malignancies in western world [1]. The NED process has recently gained considerable interest, as it may represent a mechanism underlying the complex phenomenon of $\mathrm{PCa}$ progression to an androgenindependent state frequently observed in relapsing $\mathrm{PCa}$ and associated to a life expectancy of only 15-20 months [2]. Indeed, clinical studies have shown that the number of neuroendocrine cells increases specifically in hormone-refractory tumours $[3,4]$, and this increase is correlated to poor prognosis and shorter survival time $[5,6]$. Moreover, numerous experimental in vitro and in vivo studies have shown that steroid-deprived media or animal castration promote transdifferentiation of $\mathrm{PCa}$ cells into neuroendocrine-like cells $[4,7,8]$, indicating that NED is directly associated to the hormonal status and may represent a consequence of hormone deprivation therapy in $\mathrm{PCa}$ [1].

Several lines of evidence suggest that prostatic neuroendocrine-like cells may influence the proliferation and aggressiveness of surrounding PCa cells through paracrine stimulation. Indeed, converging data from clinical and experimental studies have linked tumour cell proliferation to the presence of neuroendocrine-like cells, $[9,10]$ and the production of peptides, such as bombesin, adrenomedullin or $26 \mathrm{RFa}$, which influence tumoural cell growth $[11,12]$.

In addition to peptides, neuroendocrine cells produce different secretory proteins which may play a role in $\mathrm{PCa}$ pathophysiology. In particular, the neuroendocrine secretory proteins chromogranin $\mathrm{A}(\mathrm{CgA})$ and secretogranin II (SgII), which belong to the granin family of acidic soluble proteins, are widely distributed throughout the neuroendocrine system [13] and co-stored with hormones and neuropeptides into specialised secretory granules. Owing to its major role in the formation of secretory granules [14], SgII is crucial for the establishment of the regulated secretory pathway which represents one of the main features of the neuroendocrine phenotype allowing the storage and release of hormones and peptides [15]. Besides, SgII may serve as a precursor of bioactive peptides that could influence the activity of other cells after secretion [13]. For instance, the SgII-derived peptide secretoneurin ( $\mathrm{SN})$ acts as an angiogenic cytokine that regulates the chemotactic activity of monocytes and vascular endothelial cells [13]. Finally, it should be noted that SgII and its derived peptides such as SN and EM66 [16] are emerging as valuable and effective markers for the diagnosis and prognosis of neuroendocrine neoplasia [17]. Indeed, plasma levels of SN are elevated in androgen-independent $\mathrm{PCa}$ [18] and EM66 is now considered as a discriminating marker for benign and malignant pheochromocytoma [17].

These observations highlight the association of $\mathrm{SgII}$ with the neuroendocrine phenotype in physiological and pathophysiological conditions, prompting us to explore its potential involvement in the NED process linked to the androgen-independent progression of PCa. In the present study, we examined the intratumoural expression of SgII to assess its correlation with PCa status, and investigated the intracellular role of SgII in the establishment of the secretory activity of tumoural prostatic cells. In addition, we investigated the possible effect of the granin on PCa cell growth.

\section{Materials and methods}

\subsection{Cell culture}

The lymph node cancer prostate cells $(\mathrm{LNCaP})$ cell line, clone FGC derived from a human PCa metastasis in lymph node $\left(\mathrm{ATCC}^{\circledR} \mathrm{CRL}-1740^{\mathrm{TM}}\right.$ ) was purchased from American Type Culture Collection (ATCC, Rockville, MD). This cell line was routinely grown at $37^{\circ} \mathrm{C}$ in $5 \% \mathrm{CO}_{2}$ and used at passages $30-35$ in the present work. LNCaP cells were maintained in RPMI-1640 (Life Technologies, Saint Aubin, France) supplemented with 10\% foetal bovine serum (Lonza, Levallois, France), $2 \mathrm{mM}$ glutamine and $100 \mathrm{U} / \mathrm{ml}$ streptomycin and penicillin (Life Technologies, Saint Aubin, France). For androgen deprivation treatments, $\mathrm{LNCaP}$ cells were maintained in a steroid-reduced medium constituted of phenol red-free RPMI-1640, 5\% charcoal-stripped foetal bovine serum (Sigma), $2 \mathrm{mM}$ glutamine and $100 \mathrm{U} / \mathrm{ml}$ streptomycin and penicillin [8].

\subsection{Expression vectors and DNA transfection}

All the expression plasmids used in this study were described previously, and encode the reporters green fluorescent protein (GFP), red fluorescent protein 
(RFP) or a truncated form of embryonic alkaline phosphatase (EAP) fused to the carboxyl terminus of the following proteins: full-length human SgII (NM_003469), including its predicted 30-residue signal peptide SIG (SgII-GFP, SgII-RFP and SgII-EAP; [14,19]); SgII signal peptide alone (SIG-GFP and SIG-EAP; [19]); human neuropeptide Y (NPY; NM_000905; NPY-GFP) was a gift from R. Mains [20]. Forty-eight hours prior to transfection, LNCaP cells were split onto poly-D + L-lysine (Sigma)-coated 12-well plates (BD Biosciences, Le Pont-de-Claix, France) or onto poly-D + L-lysine-coated 4-well Lab-Tek ${ }^{\circledR}$ chamber Permanox ${ }^{\circledR}$ slides (NALGENUNC, Cergy-Pontoise, France). Cells were transfected using the MagnetoFection ${ }^{\mathrm{TM}}$ method and the LipoMag Kit (Oz Biosciences, Marseille, France), with $1 \mu \mathrm{g}$ (Lab-Tek ${ }^{\circledR}$ slides) or $2 \mu \mathrm{g}$ (12-well plates) of DNA per well and 1:3 (DNA:DreamFect ${ }^{\mathrm{TM}}$ gold reagent) and 1:1 (DNA:CombiMag reagent) ratios. Cells were placed on the Super Magnetic Plate for $15 \mathrm{~min}$, and the culture medium was replaced $5 \mathrm{~h}$ after the onset of the transfection.

\subsection{RNA extraction and quantitative polymerase chain reaction $(Q-P C R)$}

Total RNA from LNCaP cells was extracted using the NucleoSpin ${ }^{\circledR}$ RNA II kit (Macherey-Nagel, Düren, Germany) according to the manufacturer's instructions. RNA $(0.9 \mu \mathrm{g})$ was reverse transcribed during $1 \mathrm{~h}$ at $42{ }^{\circ} \mathrm{C}$ using the ImProm-II ${ }^{\mathrm{TM}}$ Reverse Transcription System for RT-PCR (Promega, Madison, WI) with $0.5 \mu \mathrm{g}$ random primers. PCR amplifications were done in duplicates using $2 \mu \mathrm{l}$ of cDNAs on the 7900 HT Fast Real-Time PCR System running the SDS 2.3 analysis software (Applied Biosystems, Courtaboeuf, France), as described previously [21]. Gene-specific forward and reverse primers were designed using the Primer Express software (Applied Biosystems, Courtaboeuf, France) as follows: 5'-TCTGCGGCGGTGTTCTG- $3^{\prime}$ and 5'-GCCGACCC AGCAAGATCA-3' $3^{\prime}(P S A), 5^{\prime}$-CGGAGAACGGGGA GGAATA-3' and $5^{\prime}$-GTCTTTGCTTCAGCCATGTT TG-3' (SGII), 5'-GATGCTGGAGTTGGATGGGA-3' and $5^{\prime}$-ACACACGGCCAGAGACACAC- $3^{\prime}$ (NSE). Q-PCR results were normalised using the multiple reference gene normalisation geNorm program (Visual Basic application tool for Microsoft Excel) and the following housekeeping genes: YWHAZ; GAPDH; HPRTI and $A L A S 1$ as previously described [21]. The resulting expression levels of the three genes of interest are further expressed as a percentage of the expression level measured before androgen deprivation.

\subsection{Immunohistochemistry}

Tissue procurement protocols were approved by the University SMBA of Fez institutional committees and were undertaken under informed consent of each patient and all of the participants. Deparaffinised sections ( $3 \mu \mathrm{m}$ thick) from 25 benign prostate hyperplasia $(\mathrm{BPH})$ and $32 \mathrm{PCa}$ of various Gleason's score were obtained from the Department of Urology of the University Hospital of Fez. All incubations were performed at room temperature. Immunohistochemical staining was performed using the UltraTech HRP streptavidin-biotin universal detection system (Immunotech, Marseille, France), or a standard avidin-biotin-peroxidase complex (Vector Laboratories, Nanterre, France). Sections were microwaved in $10 \mathrm{mM}$ citrate buffer ( $\mathrm{pH} 6$ ) for antigen retrieval and cooled in phosphate buffered saline (PBS). After endogenous peroxidase activity quenching with $3 \%$ hydrogen peroxide and tissue blocking, sections were incubated for $2 \mathrm{~h}$ with rabbit polyclonal antibody against human SgII (anti-EM66, 1:800; [16]) or mouse monoclonal antibody against $\mathrm{CgA}$ (anti-CgA, clone LK2H10, CellMarque), followed by biotinylated secondary antibody (1:400; Vector Laboratories, Nanterre, France) and the streptavidin-peroxidase reagent. Peroxidase activity was revealed with diaminobenzidine (DAB, Sigma). The slices were then counterstained for $3 \mathrm{~min}$ with haematoxylin. Observations and photomicrographs were made under a Leica Leitz light microscope.

\subsection{Photoprotein fluorescence and immunocytochemistry}

Cells were fixed for $30 \mathrm{~min}$ at room temperature with $4 \%$ paraformaldehyde in PBS, $\mathrm{pH} 7.4$, permeabilised for 10 min with $0.5 \%$ Triton X100 in PBS, and exposed to $1 \mu \mathrm{g} / \mathrm{mL}$ of the nucleic acid stain Hoechst 33258 (Polyscience, Inc., Eppelheim, Germany) for nuclei visualisation. For immunocytochemistry, permeabilised cells were treated as described previously [19], except that they were mounted in buffered Mowiol 4-88 (Polyscience, Inc., Eppelheim, Germany). Primary antibodies were a rabbit polyclonal anti-human EM66 (1:1500; [16]), a sheep polyclonal anti-TGN46 (1:2000; AbD Serotec, Colmar, France) or a mouse monoclonal antiGLUD1 antibody (1:1000; Sigma). Secondary antibodies (1:300) were Alexa Fluor IgGs from Molecular Probes (Saint Aubin, France): 488-conjugated donkey anti-rabbit, 594-conjugated donkey anti-sheep and 594-conjugated donkey anti-mouse.

\subsection{Fluorescence imaging}

Images were acquired using a Leica TCS-SP2 AOBS inverted confocal laser scanning microscope (DMIRE2; Leica Microsystems, Reuil-Malmaison, France). For each cell, $10-12$ optical $x y$ sections $(0.42 \mu \mathrm{m}$ thick $)$ along the $z$ axis were acquired with a $63 \times$ oil immersion objective (N.A. 1.4). The following excitation and emission wavelengths were used for imaging: GFP and Alexa 
Fluor 488-conjugated antibodies (green), $\lambda_{\mathrm{ex}} 488 / \lambda_{\mathrm{em}}$ $525 \pm 25 \mathrm{~nm}$; RFP and Alexa Fluor 594 antibodies (red), $\lambda_{\text {ex }} 561 \mathrm{~nm} / \lambda_{\text {em }} 650 \pm 50 \mathrm{~nm}$; Hoechst 33258 (nuclear DNA stain, blue), $\lambda_{\mathrm{ex}} 405 / \lambda_{\mathrm{em}} 445 \pm 30 \mathrm{~nm}$. Optical sections and $3 \mathrm{D}$ views were processed using Image J and/or GIMP softwares.

\subsection{Quantification of fluorescence colocalisation}

The extent of colocalisation between fluorescence signals was analysed using the ImageJ software and the JACoP colocalisation plug-in [22]. The averaged overlap coefficient $\left(R_{o}\right)$, quantitative of the degree of overlap between two fluorescent signals, was calculated for every 10-12 optical $x y$ sections along the $z$ axis of 3-5 cells.

\subsection{Protein extraction and western blotting}

Total cell lysates were prepared as described previously [23]. Proteins (50 $\mu \mathrm{g} /$ well) were separated by sodium dodecyl sulphate polyacrylamide gel electrophoresis (SDS-PAGE) on $8 \%$ polyacrylamide gels and transferred onto nitrocellulose sheets (LI-COR Biosciences, Cambridge, United Kingdom (UK)). Membranes were blocked for $30 \mathrm{~min}$ at room temperature in Odyssey ${ }^{\circledR}$ blocking buffer (LI-COR Biosciences, Cambridge, UK) and subsequently incubated at $4{ }^{\circ} \mathrm{C}$ overnight in $50 \%$ Odyssey ${ }^{\circledR}$ blocking buffer plus $0.01 \%$ Tween 20 in PBS with the following primary antibodies: a rabbit polyclonal anti-human SgII (anti-EM66, 1:2000, [16]); a rabbit polyclonal anti-human prostate-specific antigen (PSA, 1:500, Dako France); a mouse monoclonal antihuman neuron-specific enolase (NSE, 1:50, Dako France, Les Ulis, France); a mouse monoclonal antiGFP (1:1,000; Roche, Meylan, France); a mouse monoclonal anti-tubulin (1:1000; Sigma). Blots were then incubated for $30 \mathrm{~min}$ at room temperature with IRDye ${ }^{\circledR}$ 800CW donkey anti-rabbit immunoglobulin $\mathrm{G}$ ( $\mathrm{IgG})$ or IRDye $^{\circledR}$ 680RD donkey anti-mouse $\operatorname{IgG}$ at $1: 10,000$ in PBS containing $0.01 \%$ Tween 20 and $0.02 \%$ SDS. Immunoreactive bands were visualised with the Odyssey ${ }^{\circledR}$ Infrared imaging system according to the manufacturer's instructions. Quantitative analysis of immunoreactivity was done with the ImageJ software.

\subsection{Secretion assay of EAP chimeras}

Secretion experiments with cells transiently expressing the EAP fusion proteins were performed as described previously [19], with the following modifications: cells were exposed for $30 \mathrm{~min}$ to the secretagogue ionomycin ( $2 \mathrm{mM}$; Fischer BioReagents ${ }^{\circledR}$, Illkirch, France), and detection of EAP enzymatic activity was achieved on a FlexStation ${ }^{\circledR} 3$ (Molecular Devices, Sunnyvale, CA). The secretion rate of EAP chimeras was calculated as a percentage of the total EAP activity present in the cells before stimulation. Total EAP activity is the sum of the amount released plus the amount remaining in the cells.

\subsection{Cell proliferation assay}

Two days post-transfection, cells (5000 per well) were seeded in triplicate in flat bottom 96-well plates (NALGE-NUNC, Cergy-Pontoise, France). The number of viable cells was determined using the CellTiter-Blue ${ }^{\circledR}$ cell viability assay (Promega, Madison, WI) according to the manufacturer's instructions. The fluorescence indicative of the metabolic capacity of the cells was recorded on a FlexStation ${ }^{\circledR} 3\left(\lambda_{\mathrm{ex}} 560 / \lambda_{\mathrm{em}} 590 \mathrm{~nm}\right)$ after $10 \mathrm{sec}$ shaking. Cell growth rate was calculated as the percentage of fluorescence measured on day 3 post-transfection, and doubling time was calculated online (Roth V. 2006 <http://www.doubling-time.com/ compute.php>).

\subsection{Presentation of data and statistical analyses}

Values are given as the means \pm S.E. of at least duplicate determinations. In the figures, data are representative of a typical experiment repeated twice or more. Statistical correlations in immunohistochemistry experiments were performed using two-sided Fisher's exact test or $\chi^{2}$-test, and other statistical analyses were performed by analysis of variance with Dunnett's or Bonferroni's post test, using the KaleidaGraph statistical software package (Synergy Software, Reading, PA). Differences were considered significant when $p<0.05$.

\section{Results}

\subsection{SgII immunoreactivity correlates with $\mathrm{PCa}$ progression}

We first assessed SgII immunoreactivity in biopsies from patients with BPH and PCa with various Gleason's scores (Fig. 1). In BPH tissues and low grade PCa, SgII immunoreactivity was virtually absent, and only occasional faint positive staining was confined to some epithelial cells of the acini (Fig. 1A and B). In contrast, numerous SgII-positive cells were present in high grade PCa tissues (Gleason's score $>7$ ), and several carcinomatous masses were intensely labelled with anti-SgII (Fig. 1C). Quantitative analysis of 32 PCa biopsy sections (Table 1a) revealed that $82 \%$ of high grade $\mathrm{PCa}$ (Gleason's score $>7$ ) expressed the granin, while only $40 \%$ of low grade PCa (Gleason's score $<7$ ) did, thus indicating a significant correlation between SgII expression and PCa progression ( $p=0.036$, Fischer's probability test; Table 1a). Concurrently, analysis of the 32 biopsies using an antibody directed against the 
A

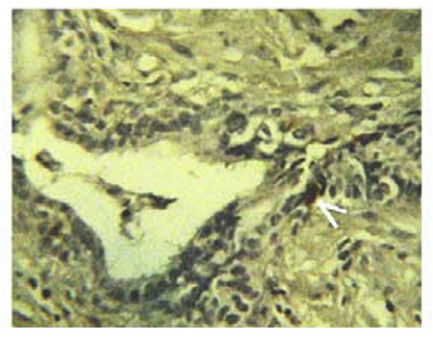

B

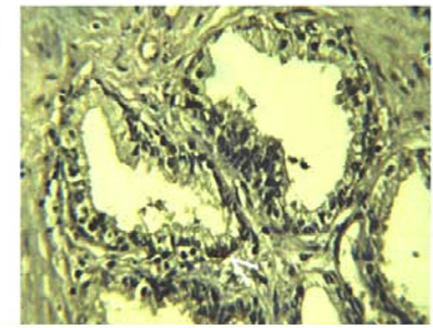

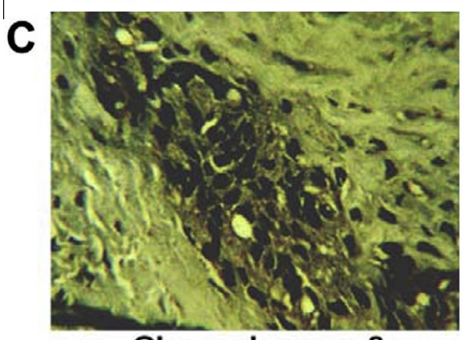

Gleason's score 8

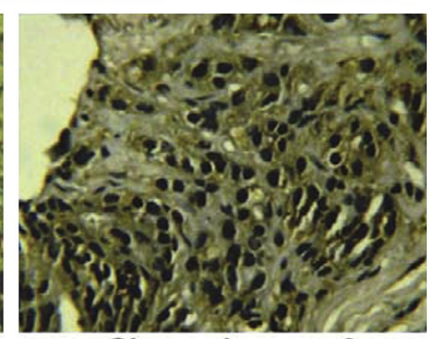

Gleason's score 9

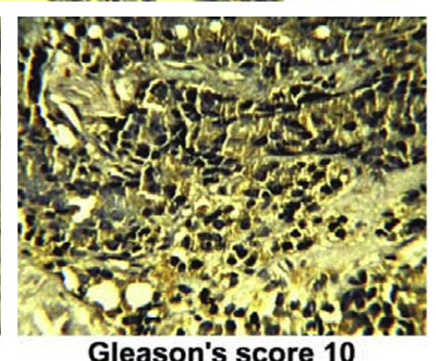

Gleason's score 10

Fig. 1. Secretogranin II (SgII) immunoreactivity in prostatic tissues. Biopsy sections from benign prostate hyperplasia (BPH) (A), low grade (Gleason's score $<7$ ) prostate cancer $(\mathrm{PCa})(\mathrm{B})$ or high grade (Gleason's scores 8, 9 and 10) PCa $(\mathrm{C})$ were processed for immunohistochemistry using an anti-EM66 (SgII-derived peptide) antibody, and the immunoreactivity was revealed with diaminobenzidine (DAB) as peroxidase-based substrate-chromogen. Representative photomicrographs are shown. Arrowheads indicate the occasional SgII-positive cells (A, B). Magnification $\times 10(\mathrm{~A}, \mathrm{~B}), \times 25(\mathrm{C})$.

Table 1

Secretogranin II (SgII) and chromogranin A (CgA) immunoreactivities in prostatic tissues.

a. Relationship between granin expression and Gleason's score

\begin{tabular}{llll}
\hline & Gleason $<7(n=10)$ & Gleason $>7(n=22)$ & $p$ value \\
\hline SgII-IR & $(-): 6(60 \%)$ & $(-): 4(18 \%)$ & $p=0.036$ \\
& $(+): 4(40 \%)$ & $(+): 18(82 \%)$ & \\
CgA-IR & $(-): 7(70 \%)$ & $(-): 6(27 \%)$ & $p=0.04$ \\
& $(+): 3(30 \%)$ & $(+): 16(73 \%)$ &
\end{tabular}

b. Correlation between $\mathrm{Cg} A$ and SgII expression in prostate cancer $(\mathrm{PCa})$

\begin{tabular}{llll}
\hline$p=0.0002$ & & CgA-IR & \\
\cline { 3 - 4 } & & $(-)(n=13)$ & $(+)(n=19)$ \\
\hline SgII-IR & $(-)(n=10)$ & $9 \underline{(69 \%)}$ & $1(5 \%)$ \\
& $(+)(n=22)$ & $4(31 \%)$ & $18 \underline{(95 \%)}$ \\
\hline
\end{tabular}

Presence $(+)$ or absence $(-)$ of SgII or CgA immunoreactivity (IR) was analysed and compared in 32 prostate adenocarcinomas of low $($ Gleason $<7)$ or high $($ Gleason $>7)$ grades. Percentages in (a) represent the proportion of positive and negative tumours for each granin. Percentages in (b) represent the proportion of tumours positive or negative for both granins, or the proportion of tumours positive for one or the other granin. Statistical significance was evaluated with a two-tailed Fisher's exact probability test $(2 \times 2$ contingency table $)$.

commonly used neuroendocrine marker $\mathrm{CgA}$ revealed an overall similar relationship between $\mathrm{CgA}$ expression and tumour grade $(p=0.049$; Fischer's probability test; Table 1a), in agreement with previous studies showing that NED is associated with $\mathrm{PCa}$ progression $[5,6]$. When comparing $\mathrm{CgA}$ and $\mathrm{SgII}$ immunoreactivities in the $32 \mathrm{PCa}$ sections, SgII exhibited a higher diagnostic value than $\mathrm{CgA}$ in high grade tumours $(82 \%$ versus $73 \%$ immunoreactive tumours, respectively). Besides, SgII was absent in $69 \%$ of the CgA-negative tumours
(Table $1 \mathrm{~b}$ ) and present in 95\% of CgA-positive biopsies (Table 1b), indicating a close relationship between the expression of the two granins in prostate tumours ( $p=0.0002$, two-tailed Fischer's exact probability test). However, few cases were positive for one granin but not the other (Table $1 \mathrm{~b}$ ), suggesting that $\mathrm{CgA}$ and $\mathrm{SgII}$ may represent complementary biomarkers in PCa.

\subsection{Androgen deprivation induces SgII expression in LNCaP cells}

Next, we wondered whether SgII abundance in high grade tumours could be recapitulated in in vitro conditions mimicking androgen-independent PCa. We therefore analysed the expression profile of SgII in the androgen-sensitive $\mathrm{LNCaP}$ cell line grown in a steroidreduced medium, mimicking androgen deprivation therapy, for 1, 4 or 7 days (Fig. 2). As expected, incubation in steroid-restricted medium provoked a rapid and significant decrease in the expression of the prostate epithelium-differentiation marker PSA, which reached very low levels after 7 days in steroid-reduced conditions ( 25 -fold decrease, $p<0.001$, Dunnett's post-test; Fig. 2A). The reduction in PSA transcript levels in LNCaP cells was accompanied by a decrease in PSA protein concentration in the same conditions (Fig. 2B), as previously reported [8]. We also monitored in these conditions NSE expression, a neuronal protein commonly used in vitro as a marker for NED [8]. As shown in Fig. 2A, LNCaP cells expressed a low level of NSE mRNA that progressively and significantly increased upon androgen starvation. A similar trend was observed for the protein (Fig. 2B), confirming the acquisition of a 
neuroendocrine phenotype by LNCaP cells in these conditions [8]. Steroid deprivation also triggered a significant $\sim 6$-fold increase in SgII mRNA levels after 7 days of treatment, $(p<0.01$, Dunnett's post-test; Fig. 2A), which was associated with an important increase $(\sim 20$ fold $)$ in SgII protein concentration (Fig. 2B). Consistently, immunocytochemical analysis showed that LNCaP cells grown in steroid-reduced medium exhibit high levels of SgII immunoreactivity as compared to LNCaP cells grown in regular medium (Fig. 2C).

\subsection{Expressing $\mathrm{SgII}$ in prostate cancer ( $\mathrm{CaP}$ ) cells induces features of NED}

Because SgII expression is up-regulated both in vivo in high grade $\mathrm{PCa}$ and in vitro in androgen-deprived LNCaP cells, we next questioned whether SgII might be an essential effector of the NED process by analysing the consequences of the expression of ectopic SgII fusion proteins in terms of neuroendocrine phenotype acquisition by LNCaP cells grown in a regular, androgencontaining medium (Figs. 3 and 4). Five days after
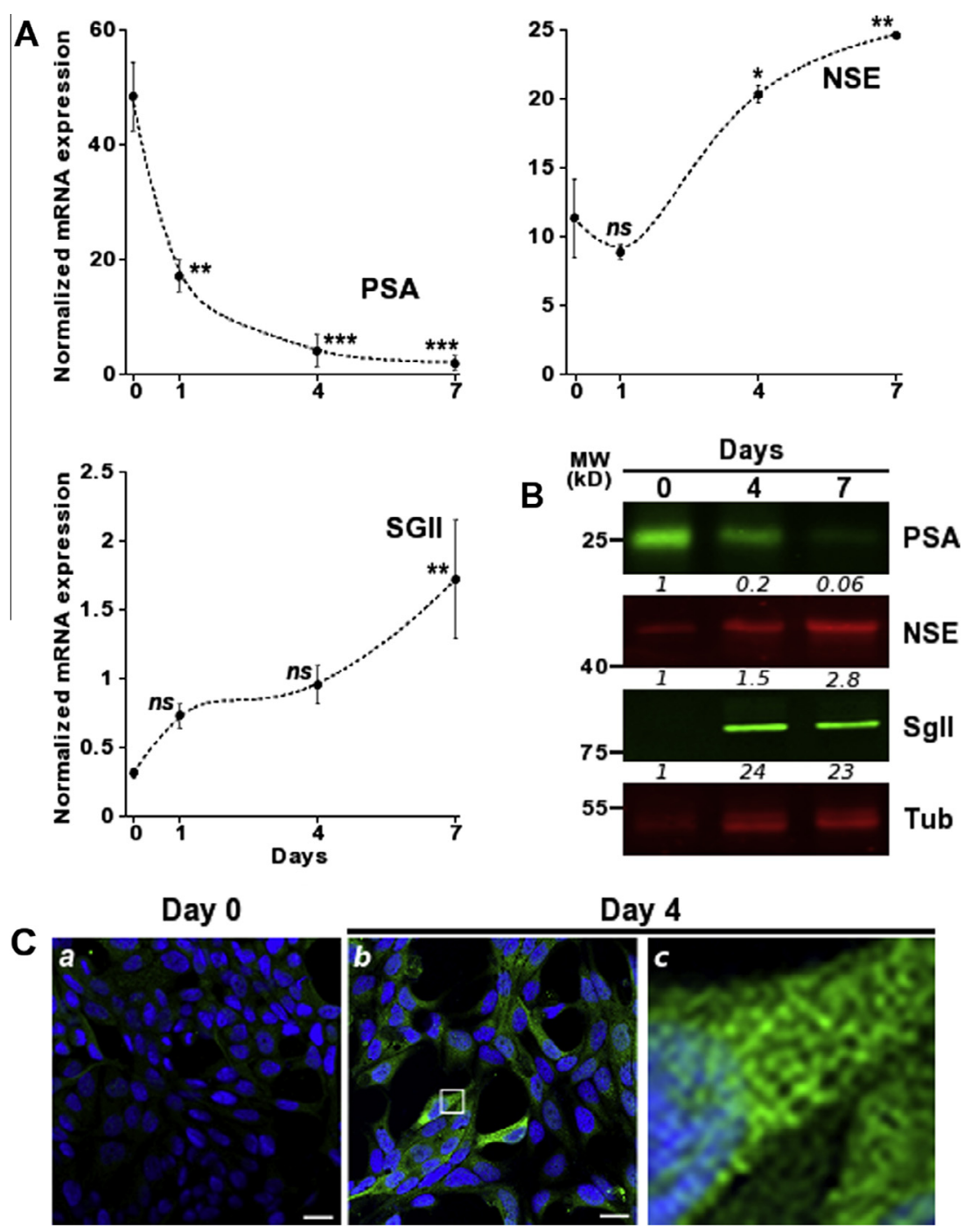

Day 4

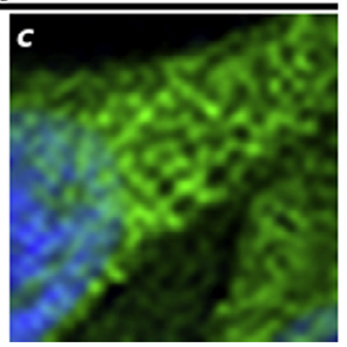

Fig. 2. Kinetics of secretogranin II (SgII) expression in LNCaP cells following androgen depletion. Total RNA and proteins from LNCaP cells grown in regular or steroid-reduced conditions during 1, 4 or 7 days were analysed by quantitative polymerase chain reaction (Q-PCR) (A) and western blot (B). (A) Normalised expression of the androgen-regulated prostate-specific antigen (PSA) and the neuroendocrine markers neuronspecific enolase (NSE) and SgII are plotted over time. Values are given as the mean \pm S.E. of at least two independent experiments. ns, $p>0.05$; ${ }^{*}, p<0.05 ;^{* *}, p<0.01,{ }^{* * *}, p<0.001$ as compared with untreated cells (day 0 ), analysis of variance (ANOVA) with Dunnett's post test. (B) Representative immunoblot ( $n=3$ ) of PSA, NSE and SgII before (0) and after 4 or 7 days of steroid removal. Tubulin (Tub) signal served as a normalisation factor. Numbers under the blots represent the normalised SgII, NSE and PSA expression levels; the signal in untreated cells was considered as 1. (C) LNCaP cells grown in regular (a) or steroid-deprived medium during 4 days (b, c) were processed for confocal microscopy. $x y$ sections stained with the anti-EM66 primary antibody (SgII, green) and Hoechst 33258 (nuclei, blue) are shown. Scale bars, $20 \mu \mathrm{m}$. (c) Magnification $(8 \times)$ of the boxed area in (b) illustrating the punctate distribution of SgII. 
transfection of LNCaP cells, the SIG-GFP (SgII signal peptide fused to GFP) and the SgII-GFP fusion proteins were correctly produced, as revealed by their expected molecular weights (Fig. 3A). Several bands with lower molecular weights and immunoreactive for both GFP and SgII were detected as well in transfected cells, suggesting the occurrence of a functional proteolytic processing of the granin in LNCaP cells (Fig. 3A).

Formation of functional secretory vesicles to establish a regulated secretory pathway represents a major feature of the neuroendocrine phenotype [15]. As shown in Fig. 3B, the SgII-GFP fusion protein transiently expressed in LNCaP cells displayed a discrete punctuate distribution of fluorescent signal throughout the cytoplasm, which is reminiscent of secretory granules in typical neuroendocrine cells $[19,24]$. In sharp contrast, the fluorescence signal of the SIG-GFP fusion protein accumulated in the perinuclear region of the transfected cells (Fig. 3B). Indeed, SIG-GFP colocalised with the Golgi marker TGN46 ( $R_{o}=0.59 \pm 0.13, n=3$; Fig. 3B), while SgII-GFP did not $\left(R_{o}=0.35 \pm 0.10, n=3\right.$; Fig. 3B). These data indicate that SgII-GFP, but not SIG-GFP, is able to induce the formation of secretory granule-like structures in $\mathrm{CaP}$ cells.
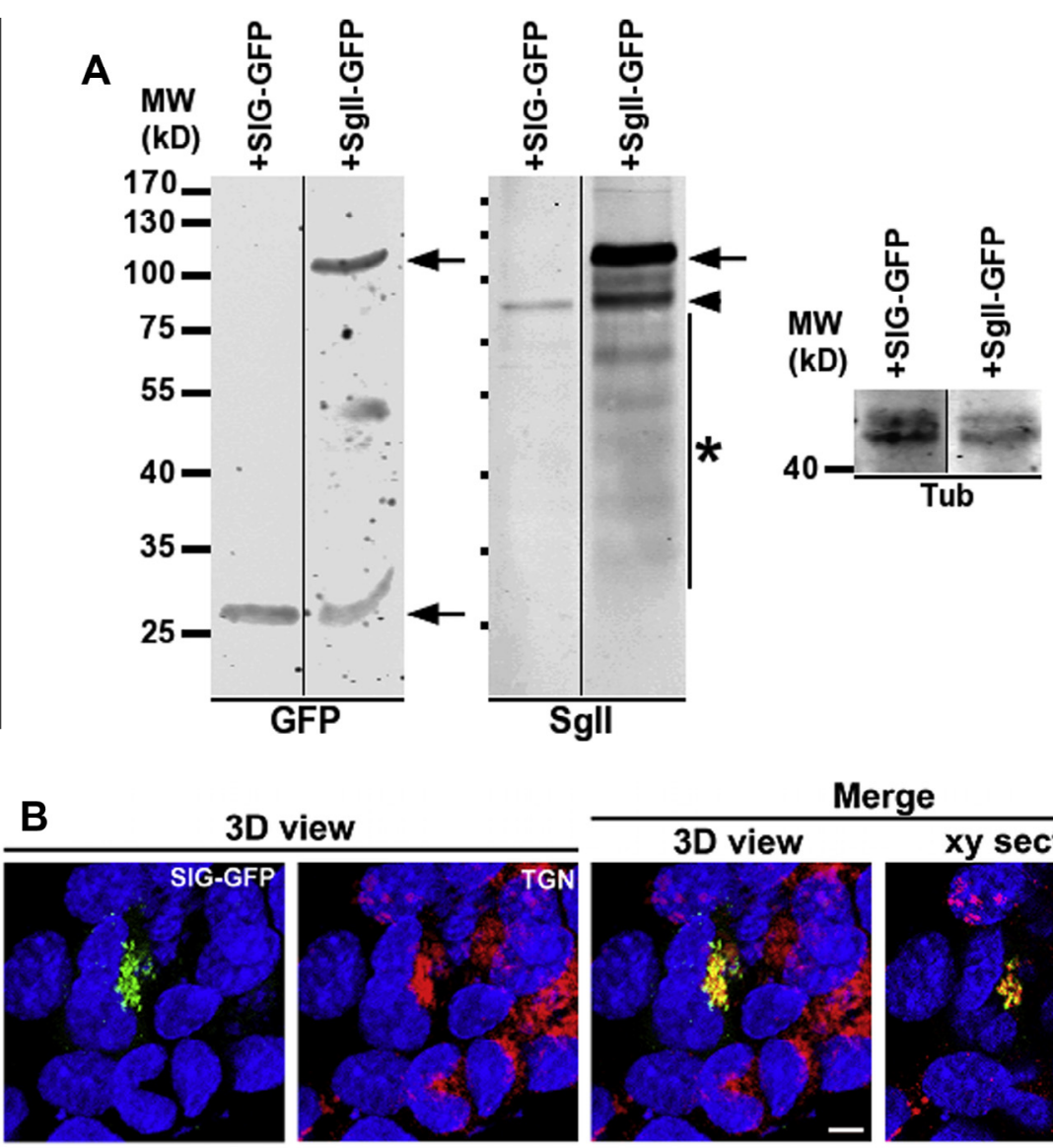

D view
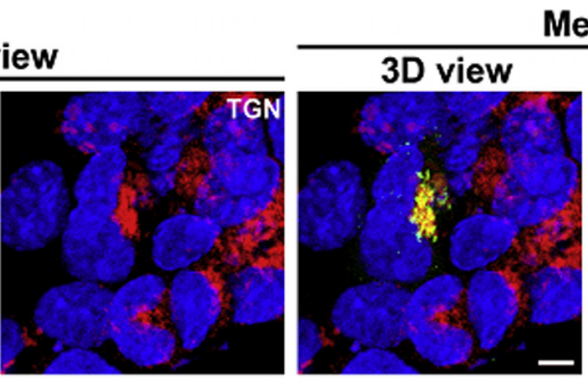

Merge
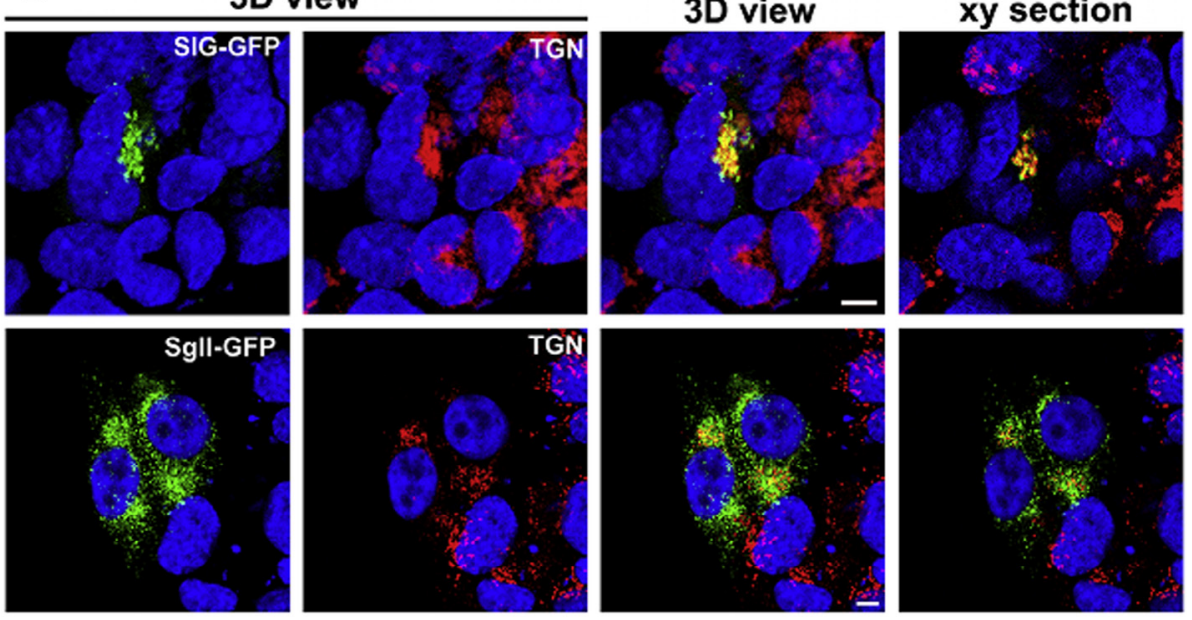

Fig. 3. Neuroendocrine differentiation features of LNCaP cells transfected with secretogranin II (SgII) fusion proteins. LNCaP cells grown in regular conditions were transfected for 5 (A) or 2 days (B) with plasmids encoding SIG-GFP or SgII-GFP fusion proteins. LNCaP cells were subjected to immunoblotting (A) or immunocytochemistry (B). Anti-GFP, anti-EM66 (SgII) or anti-tubulin (Tub; loading control) primary antibodies were used (A). Arrows indicate the full-length GFP fusion proteins $(\sim 110 \mathrm{kD}$ : SgII-GFP; $\sim 27 \mathrm{kD}$ : SIG-GFP), arrowhead indicates endogenous SgII ( $\sim 80 \mathrm{kD})$ and asterisk shows the proteolytic fragments of processed SgII. (B) Anti-TGN46 (Golgi marker) primary and Alexa Fluor 594-conjugated secondary antibodies were used. Confocal microscopy data sets were processed to generate three-dimensional (3D) volume or representative $x y$ section $(0.42 \mu \mathrm{m})$ views of the transfected cells. The distribution of the GFP chimera (green) and the endogenous TGN marker (red) was compared in merged images, and the yellow colour is indicative of colocalisation. Nuclei were visualised with Hoechst 33258 (blue). Scale bars, $5 \mu \mathrm{m}$. 


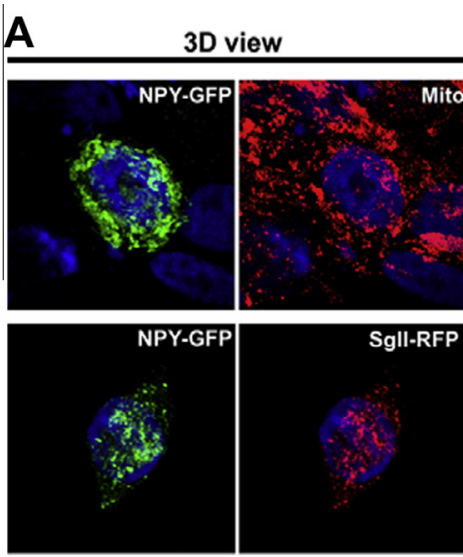

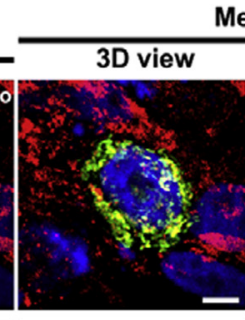

Merge
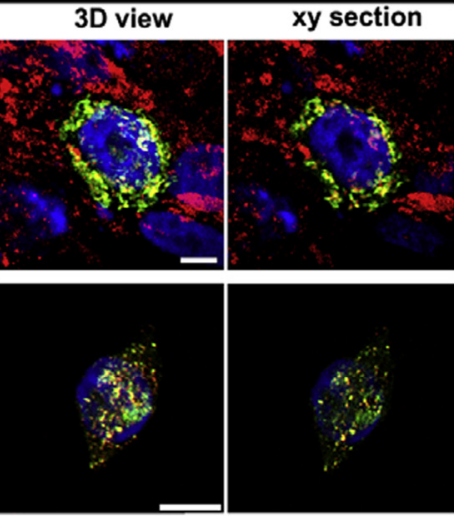
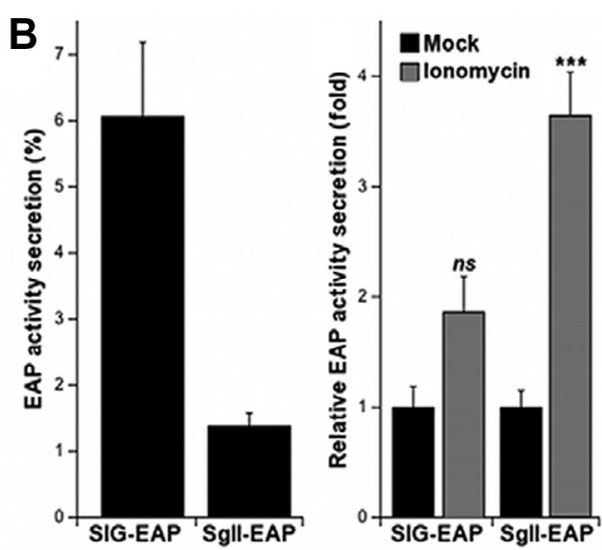

Fig. 4. Properties of secretogranin II (SgII)-containing structures in transfected LNCaP cells. (A) Cells transiently transfected with a plasmid encoding neuropeptide Y (NPY)-GFP, alone (upper panels) or together with a plasmid encoding SgII-RFP (lower panels), were processed for confocal microscopy. Anti-GLUD1 (Mito) primary antibody was used for mitochondrial staining (upper panels). Colocalisation (yellow) of NPYGFP (green) with either mitochondrial network or SgII-RFP (red) is shown in the merged images of representative 3D views or optical $x y$ sections. Nuclei were visualised with Hoechst 33258 (blue). Scale bar, $5 \mu \mathrm{m}$. (B) Cells transiently expressing SIG-EAP or SgII-EAP were exposed for 30 min to Calcium Saline Buffer alone (mock) or to $2 \mathrm{mM}$ ionomycin. Embryonic alkaline phosphatase (EAP) secretion was calculated relative to total enzymatic activity present in the cells before stimulation. Basal release of EAP (in mock) is expressed as \%EAP activity secretion in the left diagram, while secretagogue-evoked secretion of EAP is expressed relative to basal enzymatic activity release in the right diagram. Values are given as the means \pm S.E. of triplicate determinations. ns, $p>0.05 ;{ }^{* * *}, p<0.001$ as compared with basal release (mock), analysis of variance (ANOVA) with Dunnett's post test. The experiment was repeated three times with similar results.

\subsection{SgII-containing structures are competent for peptide storage and regulated release}

NPY is a prototype neuropeptide whose secretion depends on the presence of a regulated secretory pathway in neuroendocrine cells [14]. When expressed alone in LNCaP cells, NPY-GFP displayed a cluttered cytoplasmic distribution similar to that of the mitochondrial network $\left(R_{o}=0.57 \pm 0.12, n=4\right.$; Fig. 4A, upper panels), in agreement with previous studies showing the missorting of exogenous NPY to mitochondria in nonneuroendocrine cells [14]. In contrast, LNCaP cells coexpressing NPY-GFP and SgII-RFP showed a marked punctate pattern for NPY-GFP which substantially overlapped with SgII-RFP signal $\left(R_{o}=0.63 \pm 0.08\right.$, $n=3$; Fig. 4A, lower panel). This finding suggests that SgII-induced secretory granule-like structures are competent for peptide storage in prostatic cells.

A sine qua none characteristic of a functional neuroendocrine secretory pathway is the ability of secretory vesicles to release their cargo upon stimulation $[15,19,25]$. Using the EAP reporter as a highly sensitive assay to quantify the secretory activity by chemiluminescence [19], we tested the competence of SgII-containing vesicles for regulated exocytosis (Fig. 4B). In basal conditions, the release of the constitutive secretory protein SIG-EAP was elevated $(6.1 \pm 0.1 \%)$, while that of SgII-EAP chimera was low $(1.4 \pm 0.2 \%$; Fig. $4 \mathrm{~B}$, left panel), consistent with a storage of the granin, but not SIG-EAP, within secretory granule-like structures in transfected cells. Stimulation of LNCaP cells with the potent $\mathrm{Ca} 2+$ ionophore ionomycin provoked only a marginal increase in the relative release of the control SIG-EAP protein in comparison to mock treatment ( $p>0.05$, Dunnett's post test; Fig. 4B, right panel), consistent with a release of this protein through the constitutive pathway of secretion $[19,24]$. In contrast, ionomycin stimulation triggered a significant $\sim 3$.6-fold increase over basal of SgII-EAP secretion $(p<0.001$, Dunnett's post test; Fig. 4B, right panel), thus demonstrating the occurrence of a regulated secretory activity in SgII-expressing tumoural prostatic cells.

\subsection{Expression of SgII increases the proliferation of LNCaP cells in androgen-containing medium}

The effect of SgII on the growth rate of LNCaP cells was investigated in an androgen-containing medium (Fig. 5). LNCaP cells expressing the control SIG-GFP fusion protein exhibited a typical $\mathrm{LNCaP}$ growth rate, with a calculated doubling time of $\sim 50 \mathrm{~h}$ (Fig. 5) similar to that reported previously [26]. Remarkably, LNCaP cells expressing SgII-GFP exhibited a significant increase in their proliferation rate $(p<0.001$, Bonferroni's post-test; Fig. 5), with a doubling time of only $\sim 30 \mathrm{~h}$ (Fig. 5), indicating that SgII expression promotes the proliferation of PCa cells.

\section{Discussion}

The present clinical and experimental investigations are the first to show that: (i) SgII expression levels are correlated with PCa progression, (ii) SgII expression is induced by androgen deprivation, (iii) SgII triggers the 


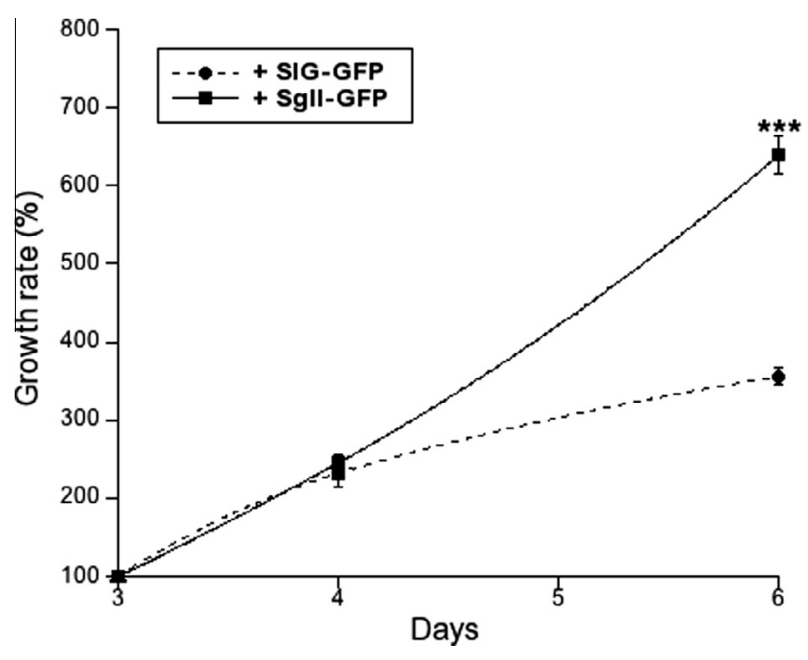

Fig. 5. Effect of secretogranin II (SgII) expression on the proliferation rate of LNCaP cells. Cells transiently transfected with plasmids encoding SIG-GFP or SgII-GFP were seeded in triplicates (5000 cells per well) $48 \mathrm{~h}$ post-transfection. Cell viability was measured after 3,4 or 6 days post-transfection. Relative cell growth rate was calculated by taking day 3 as 100 . Values are given as the means \pm S.E. of triplicate determinations. ${ }^{* * *}, p<0.001$ as compared with SIG-GFP-transfected cells, analysis of variance (ANOVA) with Bonferroni's post test. The experiment was repeated twice with similar results.

appearance of neuroendocrine features in PCa cells, and (iv) SgII promotes cancer cell proliferation. Although high circulating levels of the SgII-derived peptide SN have been previously described in hormone therapyresistant PCa [18], SgII occurrence and its possible role in $\mathrm{PCa}$ tissue have not been investigated before. Our present data revealed that $\mathrm{SgII}$ is a better indicator of PCa grade than the archetypal neuroendocrine marker $\mathrm{CgA}$ in the tested cohort. It should be noted that the value of $\mathrm{CgA}$ for the diagnosis of localised $\mathrm{PCa}[27,28]$ and for predicting time to recurrence and survival in patients with advanced $\mathrm{PCa}[3,5]$ is still a matter of debate. In fact, no other neuroendocrine marker (e.g. NSE, synaptophysin) has proved to be reliable for PCa survey [29]. The granin SgII represents a valuable tool for the diagnosis and prognosis of neuroendocrine tumours such as pheochromocytomas [17,30], but its use as a marker of other endocrine-related cancers remains infrequent. Even though our present findings need to be substantiated in a larger cohort, they strongly suggest that SgII may also represent a valuable marker that can be used alone or in combination with other neuroendocrine markers, e.g. $\mathrm{CgA}$, for the diagnosis and follow-up of PCa.

Consistent with its overexpression in androgen-independent $\mathrm{PCa}$, we found that $\mathrm{SgII}$ is induced by androgen deprivation in LNCaP cells, both at the mRNA and protein levels. Interestingly, our results show a rapid increase in SgII expression in LNCaP cells after androgen depletion, while increased $\mathrm{CgA}$ expression is only observed after long-term androgen deprivation [8], which may explain the moderate prognostic value of $\mathrm{CgA}$ in PCa. The molecular mechanism leading to the up-regulation of SgII gene expression upon androgen deprivation is not known yet but is presumably related to the activation of the protein kinase A (PKA) pathway usually observed under these conditions. Indeed, it has been shown that androgen reduction is accompanied by an increase in PKA subunit expression in LNCaP cells and PCa specimens [31], suggesting that androgen therapy-refractory tumours acquire a neuroendocrine phenotype through activation of the PKA signalling pathway. In fact, it is now well established that PKA plays a key role in the progression of $\mathrm{PCa}$ through a cross-talk with the androgen receptor [32]. The PKAmediated signalling exerts important effects on cellular growth in various cell types including prostatic cells [33], by regulating the androgen receptor activity [32] and the expression of various PKA-regulated genes involved in neuroendocrine differentiation [34]. We have previously shown that SgII gene expression is also stimulated by the cyclic adenosine monophosphate (cAMP)/ PKA pathway in the androgen-independent tumoural prostatic cells DU145 [11], suggesting that the increase in SgII levels observed in androgen-deprived LNCaP cells is likely due to activation of PKA which occurs during NED and PCa progression. Thus, PKA-mediated increase in SgII expression would contribute to the neuroendocrine differentiation and the associated implementation of a secretory activity in PCa. Using transient ectopic expression of fusion proteins in native LNCaP cells, we showed in the present study that SgII induces the formation of functional intracellular vesicles, competent for peptide storage and $\mathrm{Ca} 2+$-dependent regulated secretion. Together our data indicate that SgII could play a granulogenic function in advanced PCa. The fact that SgII is induced by androgen deprivation in the androgen-dependent LNCaP cells (the present study) or cAMP/PKA stimulation in the androgen-independent DU145 cells [11] indicates that its granulogenic role accompanies NED in PCa. Because NED in PCa cells has been shown to be associated with the formation of secretory granules [35,36] and the increase in Ca2+-dependent secretion [37], our results support the notion that SgII plays a fundamental role in NED by recapitulating key features of this process which allows the release of cancer-promoting factors. Our published [24] and unpublished (Delestre et al.) results indicate that granins such as $\mathrm{CgA}$ and $\mathrm{SgII}$ interact with lipids present in the membranes of the Golgi apparatus and with molecular motors such as myosins in order to induce the budding and formation of secretory granules. Together, these mechanistic insights into the regulated secretion from tumoural prostatic cells indicate that PKA activation could play an important role in secretory granule formation through SgII regulation during NED and may help to propose additional 
therapeutic options for advanced PCa. Such important role of PKA in NED of advanced PCa should be substantiated in future studies.

Expression of SgII led to increased proliferation of LNCaP cells, indicating that the granin confers a proliferative advantage to tumoural prostatic cells. Although the mechanism of action of SgII cannot directly be inferred from our present data, it is tempting to speculate that SgII-mediated secretory activity is responsible for a higher tumoural cell growth. In support of this hypothesis, it has been shown that neuroendocrine differentiation promotes the growth and tumourigenesis of PCa cells through secretion of peptides with mitogenic activity $[9,38]$. Therefore, SgII by promoting the formation of secretory granules containing bioactive peptides in transdifferentiated prostate cells may lead to a higher proliferation rate of adenocarcinomatous cells which are predominant in $\mathrm{PCa}$ [1]. In addition, our results indicate that $\mathrm{SgII}$ processing products are also produced by tumoural cells and could impact $\mathrm{PCa}$ cell growth. Previous studies have shown that the SgIIderived peptide SN could act as a growth factor that stimulates angiogenic processes [39]. The direct effect of SN on tumour cell growth is not known yet, but its high levels in the plasma of patients [18] suggest that this SgII-derived peptide may exert a role in $\mathrm{PCa}$ progression.

In conclusion, the present data show for the first time that $\mathrm{SgII}$ is expressed in $\mathrm{PCa}$ and that its increased levels correlate with high grade tumours. Therefore, SgII may represent a valuable clinical tool to assess PCa progression. In addition, $\mathrm{SgII}$ expression triggers a secretory activity in tumoural cells, including the appearance of secretory granules that store and release bioactive peptides, a hallmark of NED in PCa, indicating that this granin may play a pivotal role in $\mathrm{PCa}$ progression. Because the NED process is exacerbated in advanced $\mathrm{PCa}$ and the possible SgII-inducing proliferative effect, this pathway may represent a new target for therapeutic intervention.

\section{Conflict of interest statement}

None declared.

\section{Acknowledgements}

This work was supported by the ARC association for cancer research, the Conseil Régional de Haute Normandie, Inserm, (U982), the University of Rouen, and the Plateforme Régionale de Recherche en Imagerie Cellulaire de Haute-Normandie (PRIMACEN). We thank Huguette Lemonnier for her valuable technical assistance, and Pr. El Fassi Med Jamal for providing materials and helpful discussions.

\section{References}

[1] Komiya A, Suzuki H, Imamoto T, Kamiya N, Nihei N, Naya Y, et al. Neuroendocrine differentiation in the progression of prostate cancer. Int J Urol 2009;16:37-44.

[2] Nelson EC, Cambio AJ, Yang JC, Ok JH, Lara Jr PN, Evans CP. Clinical implications of neuroendocrine differentiation in prostate cancer. Prostate Cancer Prostatic Dis 2007;10:6-14.

[3] Hirano D, Okada Y, Minei S, Takimoto Y, Nemoto N. Neuroendocrine differentiation in hormone refractory prostate cancer following androgen deprivation therapy. Eur Urol 2004;45:586-92.

[4] Ismail AH, Landry F, Aprikian AG, Chevalier S. Androgen ablation promotes neuroendocrine cell differentiation in dog and human prostate. Prostate 2002;51:117-25.

[5] Berruti A, Bollito E, Cracco CM, Volante M, Ciccone G, Porpiglia F, et al. The prognostic role of immunohistochemical chromogranin a expression in prostate cancer patients is significantly modified by androgen-deprivation therapy. Prostate 2010;70:718-26.

[6] McWilliam LJ, Manson C, George NJ. Neuroendocrine differentiation and prognosis in prostatic adenocarcinoma. Br J Urol 1997;80:287-90.

[7] Marchiani S, Tamburrino L, Nesi G, Paglierani M, Gelmini S, Orlando C, et al. Androgen-responsive and -unresponsive prostate cancer cell lines respond differently to stimuli inducing neuroendocrine differentiation. Int J Androl 2010;33:784-93.

[8] Yuan TC, Veeramani S, Lin FF, Kondrikou D, Zelivianski S, Igawa $\mathrm{T}$, et al. Androgen deprivation induces human prostate epithelial neuroendocrine differentiation of androgen-sensitive LNCaP cells. Endocr Relat Cancer 2006;13:151-67.

[9] Deeble PD, Cox ME, Frierson Jr HF, Sikes RA, Palmer JB, Davidson RJ, et al. Androgen-independent growth and tumorigenesis of prostate cancer cells are enhanced by the presence of PKA-differentiated neuroendocrine cells. Cancer Res 2007;67:3663-72.

[10] Grobholz R, Griebe M, Sauer CG, Michel MS, Trojan L, Bleyl U. Influence of neuroendocrine tumor cells on proliferation in prostatic carcinoma. Hum Pathol 2005;36:562-70.

[11] Alonzeau J, Alexandre D, Jeandel L, Courel M, Hautot C, El Yamani FZ, et al. The neuropeptide 26RFa is expressed in human prostate cancer and stimulates the neuroendocrine differentiation and the migration of androgeno-independent prostate cancer cells. Eur J Cancer 2013;49:511-9.

[12] Berenguer-Daizé C, Boudouresque F, Bastide C, Tounsi A, Benyahia Z, Acunzo J, et al. Adrenomedullin blockade suppresses growth of human hormone-independent prostate tumor xenograft in mice. Clin Cancer Res 2013;19:6138-50.

[13] Montero-Hadjadje M, Vaingankar S, Elias S, Tostivint H, Mahata SK, Anouar Y. Chromogranins A and B and secretogranin II: evolutionary and functional aspects. Acta Physiol (Oxf) 2008;192:309-24.

[14] Courel M, Soler-Jover A, Rodriguez-Flores JL, Mahata SK, Elias $\mathrm{S}$, Montero-Hadjadje $\mathrm{M}$, et al. Pro-hormone secretogranin II regulates dense core secretory granule biogenesis in catecholaminergic cells. J Biol Chem 2010;285:10030-43.

[15] Malosio ML, Giordano T, Laslop A, Meldolesi J. Dense-core granules: a specific hallmark of the neuronal/neurosecretory cell phenotype. J Cell Sci 2004;117:743-9.

[16] Anouar Y, Desmoucelles C, Yon L, Leprince J, Breault L, GallotPayet N, et al. Identification of a novel secretogranin II-derived peptide $(\operatorname{SgII}(187-252))$ in the adult and fetal human adrenal glands using antibodies raised against the human recombinant peptide. J Clin Endocr Metab 1998;83:2944-51.

[17] Guerin M, Guillemot J, Thouennon E, Pierre A, El-Yamani FZ, Montero-Hadjadje M, et al. Granins and their derived peptides in 
normal and tumoral chromaffin tissue: implications for the diagnosis and prognosis of pheochromocytoma. Regul Pept 2010;165:21-9.

[18] Ischia R, Hobisch A, Bauer R, Weiss U, Gasser RW, Horninger $\mathrm{W}$, et al. Elevated levels of serum secretoneurin in patients with therapy resistant carcinoma of the prostate. J Urol 2000;163:1161-5.

[19] Courel M, Vasquez MS, Hook VY, Mahata SK, Taupenot L. Sorting of the neuroendocrine secretory protein Secretogranin II into the regulated secretory pathway: role of $\mathrm{N}$ - and C-terminal alpha-helical domains. J Biol Chem 2008;283:11807-22.

[20] El Meskini R, Jin L, Marx R, Bruzzaniti A, Lee J, Emeson RB, et al. A signal sequence is sufficient for green fluorescent protein to be routed to regulated secretory granules. Endocrinology 2001;142:864-73

[21] Thouennon E, Pierre A, Tanguy Y, Guillemot J, Manecka DL, Guerin M, et al. Expression of trophic amidated peptides and their receptors in benign and malignant pheochromocytomas: high expression of adrenomedullin RDC1 receptor and implication in tumoral cell survival. Endocr Relat Cancer 2010;17:637-51.

[22] Bolte S, Cordeliere FP. A guided tour into subcellular colocalization analysis in light microscopy. J Microsc 2006;224: $213-32$.

[23] Courel M, Rodemer C, Nguyen ST, Pance A, Jackson AP, O'Connor DT, et al. Secretory granule biogenesis in sympathoadrenal cells: identification of a granulogenic determinant in the secretory prohormone chromogranin A. J Biol Chem 2006;281:38038-51.

[24] Montero-Hadjadje M, Elias S, Chevalier L, Benard M, Tanguy Y, Turquier V, et al. Chromogranin A promotes peptide hormone sorting to mobile granules in constitutively and regulated secreting cells: role of conserved N- and C-terminal peptides. J Biol Chem 2009:284:12420-31.

[25] Elias S, Delestre C, Ory S, Marais S, Courel M, VazquezMartinez R, et al. Chromogranin A induces the biogenesis of granules with calcium- and actin-dependent dynamics and exocytosis in constitutively secreting cells. Endocrinology 2012; 153:4444-56.

[26] Horoszewicz JS, Leong SS, Kawinski E, Karr JP, Rosenthal H, Chu TM, et al. LNCaP model of human prostatic carcinoma. Cancer Res 1983;43:1809-18.

[27] De Nunzio C, Albisinni S, Presicce F, Lombardo R, Cancrini F, Tubaro A. Serum levels of chromogranin A are not predictive of high-grade, poorly differentiated prostate cancer: results from an Italian biopsy cohort. Int Braz J Urol 2013;39:793-9.
[28] Masieri L, Lanciotti M, Gontero P, Marchioro G, Mantella A, Zaramella S, et al. The prognostic role of preoperative chromogranin A expression in prostate cancer after radical prostatectomy. Arch Ital Urol Androl 2012;84:17-21.

[29] Ather MH, Abbas F, Faruqui N, Israr M, Pervez S. Correlation of three immunohistochemically detected markers of neuroendocrine differentiation with clinical predictors of disease progression in prostate cancer. BMC Urol 2008;8:21.

[30] Guillemot J, Anouar Y, Montero-Hadjadje M, Grouzmann E, Grumolato L, Roshmaninho-Salgado J, et al. Circulating EM66 is a highly sensitive marker for the diagnosis and follow-up of pheochromocytoma. Int J Cancer 2006;118:2003-12.

[31] Kvissel AK, Ramberg H, Eide T, Svindland A, Skålhegg BS, Taskén KA. Androgen dependent regulation of protein kinase A subunits in prostate cancer cells. Cell Signal 2007;19:401-9.

[32] Sarwar M, Sandberg S, Abrahamsson PA, Persson JL. Protein kinase A (PKA) pathway is functionally linked to androgen receptor (AR) in the progression of prostate cancer. Urol Oncol 2014;32, 25.e1-12.

[33] Merkle D, Hoffmann R. Roles of cAMP and cAMP-dependent protein kinase in the progression of prostate cancer: cross-talk with the androgen receptor. Cell Signal 2011;23:507-15.

[34] Park MH, Lee HS, Lee CS, You ST, Kim DJ, Park BH, et al. P21-Activated kinase 4 promotes prostate cancer progression through CREB. Oncogene 2013;32:2475-82.

[35] Bang YJ, Pirnia F, Fang WG, Kang WK, Sartor O, Whitesell L, et al. Terminal neuroendocrine differentiation of human prostate carcinoma cells in response to increased intracellular cyclic AMP. Proc Natl Acad Sci USA 1994;91:5330-4.

[36] Cox ME, Deeble PD, Lakhani S, Parsons SJ. Acquisition of neuroendocrine characteristics by prostate tumor cells is reversible: implications for prostate cancer progression. Cancer Res 1999;59:3821-30.

[37] Gackiere F, Bidaux G, Delcourt P, Van Coppenolle F, Katsogiannou M, Dewailly E, et al. CaV3.2 T-type calcium channels are involved in calcium-dependent secretion of neuroendocrine prostate cancer cells. J Biol Chem 2008;283:10162-73.

[38] Hafiz S, Dennis JC, Schwartz D, Judd R, Tao YX, Khazal K, et al. Expression of melanocortin receptors in human prostate cancer cell lines: MC2R activation by ACTH increases prostate cancer cell proliferation. Int J Oncol 2012;41:1373-80.

[39] Albrecht-Schgoer K, Schgoer W, Holfeld J, Theurl M, Wiedemann D, Steger C, et al. The angiogenic factor secretoneurin induces coronary angiogenesis in a model of myocardial infarction by stimulation of vascular endothelial growth factor signaling in endothelial cells. Circulation 2012;126:2491-501. 